\title{
入出力誤差分散推定を用いた 多入力多出カシステムのパラメータ同定法*
}

小松 信雄

\author{
An Identification Method for Multiple-Input Multiple-Output \\ Linear Systems with Estimation of Variance of Input-Output \\ Noises*
}

Nobuo Komatsu ${ }^{\dagger}$

\begin{abstract}
A new algorithm is proposed to estimate parameters of MIMO (Multiple-Input Multiple-Output) system. The algorithm is an expansion of the previously proposed algorithm by the author for SISO (Single-Input Single-Output) system and MISO (Multiple-Input Single-Output) system. Those algorithms use an iterative calculation and they consist of two parts. One part is the estimation of the system parameters using the variances of errors. The other part is the estimation of the variances of errors using the system parameters. The estimation of the system parameters is one of the least squares identification algorithms using eigenvector. The variances of errors are estimated by solving linear simultaneous equations derived from the transfer functions of the system. Some simulations show the effectiveness of the proposed method.
\end{abstract}

\section{1. 緒論}

本論文では，すでに発表された入出力誤差分散推定 を用いた一入力一出力システム（以下では SISO システ ムとする）および, 多入力一出力システム（以下では MISO システムとする）に対するパラメータ同定法 $[1,2]$ の多入力多出力システム（以下では MIMO システムと する）への拡張について述べる.

システム同定問題に対して現在まで多くの研究が行わ れている. 最小二乗法, 補助変数法, 部分空間法などが 提案されさまざまな方法が知られている $[3-7]$. また，近 年はロバスト同定などについての研究が進んでいる.

本論文が対象とするシステムのモデルは, 入出力誤差 モデルを対象としたものであり, 統計学の分野では変数 誤差モデル (errors-in-variables model[8]) とよばれる. このようなモデルに対する同定法としては，古典的同定 法の最小二乗法の改良である固有べクトル法 [7] があり， 提案手法もその一種といえる.

一般に固有ベクトル法は, 各時間における入出力の計 測值が与えられたとき，それらによって決まる行列の固

* 原稿受付 2015 年 7 月 15 日

$\dagger$ 大阪工業大学 情報科学部 Faculty of Information Science, Osaka Institute of Technology; 1-79-1 Kitayama, Hirakata city, Osaka 573-0196, JAPAN

Key Words: system identification, eigenvector method, digital transfer function.
有べクトルを求めることによって解が得られる。ただし， 入出力誤差の分散について, 少なくともそれらの比の值 が必要である。そこで, 固有べクトルを用いて誤差の分 散からパラメー夕推定值を求め, パラメータ推定值から 誤差分散を求めることを繰り返す同定法が考えられる. 入出力誤差の分散の值を求める方法として, 入出力デー 夕の相関関数を用いるものや, 同定次数を変化させて得 られる誤差分散に関する連立非線形方程式を用いるもの などが提案されている [8-10]. しかし，それらは計算量 が多い方法であったり, 解が求めにくい方法であるなど の問題点がある.

それに対し著者は，一入力一出力システムに対し，入 出力関係から誤差分散についての線形な連立方程式を導 出することにより，比較的容易に誤差分散の值を求める 方法を提案した 11$]$. さらに, MISO システムを対象とし て, 伝達関数のパラメータから計算される合成パラメー 夕を導出し，それによりSISO システムの場合と同様に パラメータ推定を行う同定法を提案した $[2]$. そして, 各 出力とその要素への入力の組合せに対して, MISO シス テム同定を行うことにより, MIMO システムの同定を行 えることが指摘されている。

そこで本論文では，MISO システムに対する同定法を 拡張した MIMO システムの同定アルゴリズムを提案す る。また, すでに提案した同定法では, 固有べクトル法 を用いる際の重み行列の計算において, 誤差の分散が 0 
に近くなると，計算機の計算精度上好ましくない場合が あるため，誤差の分散值の下限を決め，計算ループが止 まらないように工夫がされている。しかし，下限の值を 計算能力に応じて適切に決める必要がある。そこで本論 文では，新しい重み行列を定義することにより，下限を 設定する必要がない計算法を実現している.

なお，本同定アルゴリズムは，合成パラメータと誤差 分散の相互を繰り返し求める計算ループと, 収束後の合 成パラメータから伝達関数パラメータを求める計算から なっている.

まず，対象とする MIMO システムについて述べ，伝 達関数パラメータから作られる合成パラメータを定義す る。合わせて, 合成パラメータから伝達関数パラメータ を決定する方法を示す，つぎに，同定アルゴリズムを示 し, 誤差分散の推定值から合成パラメータを求める方法, 合成パラメータから誤差分散の推定值を求める方法, 誤 差分散推定計算に必要な係数を再帰的な関数によって求 める方法を順に示す.

さらに，本方式の有効性を確認するために行った，二 入力二出力システムに対するシミュレーションの内容, およびその結果について述べる.

\section{MIMO システムの同定}

\section{1 対象とするシステム}

提案する同定アルゴリズムは，Fig. 1 に示されるよう な MIMO システムに対するものである.

各 $G_{i j}(z)$ で示される要素に対する入力および出力 を, $\xi_{j, t}, \eta_{i, t}(i=1, \ldots, r ; j=1, \ldots, m ; t=1, \ldots, N)$ とする. そして $, y_{i, t}, u_{j, t}, \eta_{i, t}, \xi_{j, t}$ を $z$ 変換したものをそれぞれ $y_{i}(z), u_{j}(z), \eta_{i}(z), \xi_{j}(z)$ とする.さらに, $u_{j, t}, \eta_{i, t}$ に, 互 いに無相関な正規白色雑音 $\Delta u_{j, t}, \Delta y_{i, t}$ が誤差として加え られ，それらの分散を $\sigma_{u_{j}}^{2}, \sigma_{y_{i}}^{2}$ とする. ただし， $\sigma_{u_{j}}^{2}, \sigma_{y_{i}}^{2}$ の 值は未知とする. なお, $t \leq 0$ に対して, $y_{i, t}, u_{j, t}, \eta_{i, t}, \xi_{j, t}$ がすべて 0 とする.

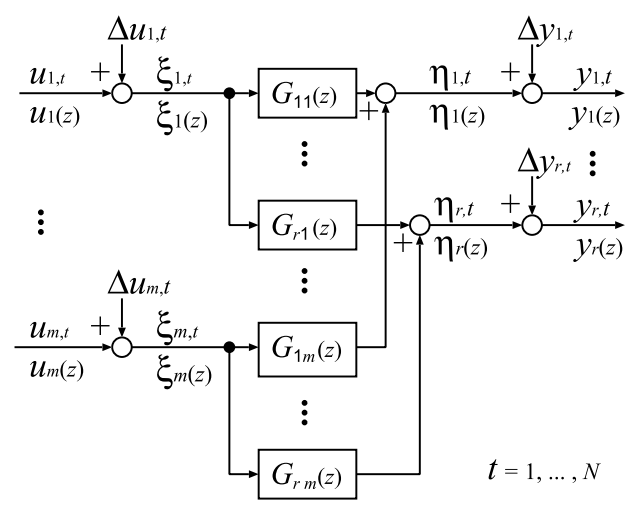

Fig. 1 MIMO-system

Fig. 1 における各伝達関数 $G_{i j}(z)$ の次数を $n_{i j}$ とし, 既知とする。また, 伝達関数を,

$$
\begin{aligned}
G_{i j}(z) & =\frac{N_{i j}(z)}{D_{i j}(z)} \\
N_{i j}(z) & =\sum_{k=1}^{n_{i j}} b_{i j, k} z^{-k} \\
D_{i j}(z) & =1+\sum_{k=1}^{n_{i j}} a_{i j, k} z^{-k}
\end{aligned}
$$

とする。このとき，

$$
\eta_{i}(z)=\sum_{j=1}^{m} G_{i j}(z) \xi_{j}(z)
$$

となる。

まず(4)式，(1)式より,

$$
\eta_{i}(z)-\sum_{j=1}^{m} \frac{N_{i j}(z)}{D_{i j}(z)} \xi_{j}(z)=0
$$

となる。

ここで, (5) 式第 2 項の分母である $D_{i j}(z)$ を消去す ると,

$$
\begin{aligned}
& \left(\prod_{k=1}^{m} D_{i k}(z)\right) \eta_{i}(z) \\
& -\sum_{j=1}^{m}\left(\prod_{k=1}^{m} D_{i k}(z)\right) \frac{N_{i j}(z)}{D_{i j}(z)} \xi_{j}(z)=0
\end{aligned}
$$

となる.これにより，(6) 式第 2 項の分母である $D_{i j}(z)$ は, $\prod_{k=1}^{m} D_{i k}(z)$ の積の項の一つと相殺される.つぎに,

$$
\begin{aligned}
\boldsymbol{z} & =\left[z^{-1}, \cdots, z^{-n_{i}}\right]^{T} \\
n_{i} & =\sum_{j=1}^{m} n_{i j}
\end{aligned}
$$

とする。

さらに, $a_{i j, k}, b_{i j, k}$ から作られる合成パラメータ $\alpha_{i, k}$, $\beta_{i j, k}, \quad\left(k=1, \ldots, n_{i}\right)$ を考え, 合成パラメータベクトル $\boldsymbol{\alpha}_{i}, \boldsymbol{\beta}_{i j}$ を,

$$
\begin{aligned}
\boldsymbol{\alpha}_{i} & =\left[\alpha_{i, 1}, \cdots, \alpha_{i, n_{i}}\right]^{T} \in \mathbf{R}^{n_{i}} \\
\boldsymbol{\beta}_{i j} & =\left[\beta_{i j, 1}, \cdots, \boldsymbol{\beta}_{i j, n_{i}}\right]^{T} \in \mathbf{R}^{n_{i}}
\end{aligned}
$$

とし,

$$
\begin{aligned}
1+\boldsymbol{\alpha}_{i}^{T} \boldsymbol{z} & =\prod_{k=1}^{m} D_{i k}(z) \\
\boldsymbol{\beta}_{i j}^{T} \boldsymbol{z} & =\left(\prod_{k=1}^{m} D_{i k}(z)\right) \frac{N_{i j}(z)}{D_{i j}(z)} \\
& =N_{i j}(z) \prod_{k \neq j} D_{i k}(z)
\end{aligned}
$$

が満たされるように $\alpha_{i, k}, \beta_{i j, k}$ を決める。

一方, 伝達関数パラメータベクトル $\boldsymbol{a}_{i j}, \boldsymbol{b}_{i j}$ を, 


$$
\begin{aligned}
\boldsymbol{a}_{i j} & =\left[a_{i j, 1}, \cdots, a_{i j, n_{i j}}\right]^{T} \in \mathbf{R}^{n_{i j}} \\
\boldsymbol{b}_{i j} & =\left[b_{i j, 1}, \cdots, b_{i j, n_{i j}}\right]^{T} \in \mathbf{R}^{n_{i j}}
\end{aligned}
$$

とする．

ここで, 合成パラメータベクトルの推定值 $\hat{\boldsymbol{\alpha}}_{i}, \hat{\boldsymbol{\beta}}_{i j}$ が 与えられたとき， $\boldsymbol{a}_{i j}, \boldsymbol{b}_{i j}$ の推定值 $\hat{\boldsymbol{a}}_{i j}, \hat{\boldsymbol{b}}_{i j}$ を求める方法 について述べる。ただし，MISO に対する同定法 [2] と 共通であるので，詳細は省略する。

まず，(11)，(12)式より，

$$
\left(\boldsymbol{\beta}_{i j}^{T} \boldsymbol{z}\right) D_{i j}(z)=\left(1+\boldsymbol{\alpha}_{i}^{T} \boldsymbol{z}\right) N_{i j}(z)
$$

が得られる。 そこで，係数比較を行い，パラメー夕間の 関係式を求めることにより, 次式が導出される [2].

$$
\left[\boldsymbol{\beta}_{i j}^{\prime}, M_{1}\right]\left[\begin{array}{c}
1 \\
\boldsymbol{a}_{i j}
\end{array}\right]=\left[\boldsymbol{\alpha}_{i j}^{\prime}, M_{2}\right]\left[\begin{array}{c}
0 \\
\boldsymbol{b}_{i j}
\end{array}\right]
$$

ただし，

$$
\begin{aligned}
& \boldsymbol{\alpha}_{i j}^{\prime}=\left[\begin{array}{llllll}
\alpha_{i, 1} & \cdots & \alpha_{i, n_{i}} & 0 & \cdots & 0
\end{array}\right]^{T} \in \mathbf{R}^{n_{i}+n_{i j}} \\
& \boldsymbol{\beta}_{i j}^{\prime}=\left[\begin{array}{llllll}
\beta_{i j, 1} & \cdots & \beta_{i j, n_{i}} & 0 & \cdots & 0
\end{array}\right]^{T} \in \mathbf{R}^{n_{i}+n_{i j}}
\end{aligned}
$$

$$
M_{1}=\left[\begin{array}{cccc}
0 & 0 & \cdots & 0 \\
\beta_{i j, 1} & 0 & \ddots & \vdots \\
\vdots & \ddots & \ddots & 0 \\
\vdots & & \ddots & 0 \\
\vdots & & & \beta_{i j, 1} \\
\beta_{i j, n_{i}} & & & \vdots \\
0 & \ddots & & \vdots \\
\vdots & \ddots & \ddots & \vdots \\
0 & \cdots & 0 & \beta_{i j, n_{i}}
\end{array}\right] \in \mathbf{R}^{\left(n_{i}+n_{i j}\right) \times n_{i j}}
$$

$$
M_{2}=\left[\begin{array}{cccc}
1 & 0 & \cdots & 0 \\
\alpha_{i, 1} & 1 & \ddots & \vdots \\
\vdots & \ddots & \ddots & 0 \\
\vdots & & \ddots & 1 \\
\vdots & & & \alpha_{i, 1} \\
\alpha_{i, n_{i}} & & & \vdots \\
0 & \ddots & & \vdots \\
\vdots & \ddots & \ddots & \vdots \\
0 & \cdots & 0 & \alpha_{i, n_{i}}
\end{array}\right] \in \mathbf{R}^{\left(n_{i}+n_{i j}\right) \times n_{i j}}
$$

とする.

したがって

$$
\boldsymbol{\beta}_{i j}^{\prime}+M_{1} \boldsymbol{a}_{i j}=M_{2} \boldsymbol{b}_{i j}
$$

となる.ここで,

$$
\begin{aligned}
\boldsymbol{p}_{G i j} & =\left[-\boldsymbol{a}_{i j}^{T}, \boldsymbol{b}_{i j}^{T}\right]^{T} \\
M & =\left[M_{1}, M_{2}\right] \in \mathbf{R}^{\left(n_{i}+n_{i j}\right) \times 2 n_{i j}}
\end{aligned}
$$

とすると，

$$
\boldsymbol{\beta}_{i j}^{\prime}=M \boldsymbol{p}_{G i j}
$$

が得られる.

そこで，この式の $M$ および $\boldsymbol{\beta}_{i j}^{\prime}$ を, $\hat{\boldsymbol{\alpha}}_{i}, \hat{\boldsymbol{\beta}}_{i j}$ から (23) 式および(18) 式，(19) 式，(20) 式を用いて構成し, $\boldsymbol{p}_{G i j}$ について解く. ただし, $M$ の行数 $\left(=n_{i}+n_{i j}\right)$ は列 数 $\left(=2 n_{i j}\right)$ 以上であり, また $M, \boldsymbol{\beta}_{i j}^{\prime}$ の要素には誤差が 含まれると考えられるので, 推定值 $\hat{\boldsymbol{p}}_{G_{i j}}$ を次のように 最小二乗法を用いて求める.

$$
\hat{\boldsymbol{p}}_{G_{i j}}=\left(M^{T} M\right)^{-1} M^{T} \boldsymbol{\beta}_{i j}^{\prime}
$$

以上より， $\hat{\boldsymbol{\alpha}}_{i}, \hat{\boldsymbol{\beta}}_{i j}$ から，(18) 式，(19)式，(20) 式を用 いて， $\boldsymbol{\beta}_{i j}^{\prime}, M_{1}, M_{2}$ を作り，(23) 式を用いて $M$ を構成し， (25) 式を用いると $\hat{\boldsymbol{p}}_{G_{i j}}$ が求められ, 伝達関数 $G_{i j}(z)$ の パラメータベクトルの推定值 $\hat{\boldsymbol{a}}_{i j}, \hat{\boldsymbol{b}}_{i j}$ が求められること が示された。

つぎに合成パラメータベクトルを, システムの入出力 データから推定する方法について述べる。まず，(6) 式 より,

$$
\left(1+\boldsymbol{\alpha}_{i}^{T} \boldsymbol{z}\right) \eta_{i}(z)-\sum_{j=1}^{m} \boldsymbol{\beta}_{i j}^{T} \boldsymbol{z} \xi_{j}(z)=0
$$

となる.ここで，

$$
\begin{aligned}
\boldsymbol{\beta}_{i} & =\left[\boldsymbol{\beta}_{i 1}^{T}, \cdots, \boldsymbol{\beta}_{i m}^{T}\right]^{T} \in \mathbf{R}^{m n_{i}} \\
\boldsymbol{\xi}_{i}(z) & =\left[\boldsymbol{z}^{T} \xi_{1}(z), \cdots, \boldsymbol{z}^{T} \xi_{m}(z)\right]^{T} \in \mathbf{C}^{m n_{i}}
\end{aligned}
$$

とすると，

$$
\left(1+\boldsymbol{\alpha}_{i}^{T} \boldsymbol{z}\right) \eta_{i}(z)-\boldsymbol{\beta}_{i}^{T} \boldsymbol{\xi}_{i}(z)=0
$$

となる.さらに，

$$
\begin{aligned}
\boldsymbol{\eta}_{i, t} & =\left[\eta_{i, t-1}, \cdots, \eta_{i, t-n_{i}}\right]^{T} \in \mathbf{R}^{n_{i}} \\
\boldsymbol{\xi}_{i, t} & =\left[\boldsymbol{\xi}_{i 1, t}^{T}, \cdots, \boldsymbol{\xi}_{i m, t}^{T}\right]^{T} \in \mathbf{R}^{m n_{i}} \\
\boldsymbol{\xi}_{i j, t} & =\left[\xi_{j, t-1}, \cdots, \xi_{j, t-n_{i}}\right]^{T} \in \mathbf{R}^{n_{i}}
\end{aligned}
$$

とすると，入出力デー夕の関係は次式のように表される.

$$
\left[\eta_{i, t}, \boldsymbol{\eta}_{i, t}^{T},-\boldsymbol{\xi}_{i, t}^{T}\right]\left[\begin{array}{r}
1 \\
\boldsymbol{\alpha}_{i} \\
\boldsymbol{\beta}_{i}
\end{array}\right]=0
$$

そこで,

$$
\begin{aligned}
n_{m i} & =n_{i}+m n_{i}+1 \\
\boldsymbol{q}_{i, t} & =\left[\eta_{i, t}, \boldsymbol{\eta}_{i, t}^{T},-\boldsymbol{\xi}_{i, t}^{T}\right]^{T} \in \mathbf{R}^{n_{m i}} \\
\boldsymbol{p}_{i} & =\left[1, \boldsymbol{\alpha}_{i}^{T}, \boldsymbol{\beta}_{i}^{T}\right]^{T} \in \mathbf{R}^{n_{m i}}
\end{aligned}
$$


とすると次式が得られ， $\boldsymbol{q}_{i, t}$ と $\boldsymbol{p}_{i}$ は直交することがわ かる。

$$
\boldsymbol{q}_{i, t}^{T} \boldsymbol{p}_{i}=0
$$

\section{2 同定アルゴリズム}

まず，合成パラメータからなるベクトル $\boldsymbol{\alpha}_{i}, \boldsymbol{\beta}_{i} よ り$ 作 られる $\boldsymbol{p}_{i}$ の推定值を,

$$
\hat{\boldsymbol{p}}_{i}=\left[1, \hat{\boldsymbol{\alpha}}_{i}^{T}, \hat{\boldsymbol{\beta}}_{i}^{T}\right]^{T}
$$

とし， $\sigma_{u_{j}}^{2}, \sigma_{y_{i}}^{2}$ の推定值を $\hat{\sigma}_{u_{j}}^{2}, \hat{\sigma}_{y_{i}}^{2}$ とする. そこで, つ ぎのような同定アルゴリズムを提案する.

$\mathrm{S} 1 \hat{\sigma}_{u_{j}}^{2}=\hat{\sigma}_{y_{i}}^{2}=1(j=1, \ldots, m ; i=1, \ldots, r)$ として, 計測 データから $\hat{\boldsymbol{p}}_{i}$ を求める.

S2 $\hat{\boldsymbol{p}}_{i}(i=1, \ldots, r)$ を用いて計測データから $\hat{\sigma}_{u_{j}}^{2}, \hat{\sigma}_{y_{i}}^{2}$ を 求める.

S3 $\hat{\sigma}_{u_{j}}^{2}, \hat{\sigma}_{y_{i}}^{2}$ を用いて計測データから, つぎのループの $\hat{\boldsymbol{p}}_{i}$ を求める.

$\mathrm{S} 4 \mathrm{~S} 2, \mathrm{~S} 3$ を, $\hat{\boldsymbol{p}}_{i}$ の值が収束判定条件を満たすまで繰 り返す.

S5 $\hat{\boldsymbol{p}}_{i}$ から， $\hat{\boldsymbol{\alpha}}_{i}, \hat{\boldsymbol{\beta}}_{i}$ が決まるので，それらを用いて伝 達関数 $G_{i j}(z)$ のパラメータベクトル $\boldsymbol{a}_{i j}, \boldsymbol{b}_{i j}$ の推 定值 $\hat{\boldsymbol{a}}_{i j}, \hat{\boldsymbol{b}}_{i j}$ を求める.

なお，S4における収束判定条件は， $\ell$ 回目のループでの 推定パラメータ $\hat{\boldsymbol{p}}_{i, \ell}$ に対して,

$$
\begin{aligned}
& \hat{\boldsymbol{p}}_{i, \ell}=\left[1, \hat{\boldsymbol{p}}_{i, \ell}^{\prime}{ }_{i}^{T}\right]^{T} \\
& \hat{\boldsymbol{p}}_{\ell}^{\prime}=\left[\hat{\boldsymbol{p}}_{1, \ell}^{\prime}, \cdots, \hat{\boldsymbol{p}}_{r, \ell}^{\prime T}\right]^{T}
\end{aligned}
$$

として, ある打ち切り判定係数 $\mu$ を決め, 次式で与える.

$$
\frac{\left\|\hat{\boldsymbol{p}}_{\ell}^{\prime}-\hat{\boldsymbol{p}}_{\ell-1}^{\prime}\right\|}{\left\|\hat{\boldsymbol{p}}_{\ell}^{\prime}\right\|} \leq \mu
$$

\section{$2.3 \hat{\sigma}_{u_{j}}^{2}, \hat{\sigma}_{y_{i}}^{2}$ を用いて $\hat{\boldsymbol{p}}_{i}$ を求める方法}

同定アルゴリズムの $\mathrm{S} 1, \mathrm{~S} 3$ における， $\sigma_{u_{j}}^{2}, \sigma_{y_{i}}^{2}$ を用 いて $\hat{p}_{i}$ を求める方法について述べる.

まず,

$$
\begin{aligned}
& y_{i, t}=\eta_{i, t}+\Delta y_{i, t} \\
& \xi_{j, t}=u_{j, t}+\Delta u_{j, t}
\end{aligned}
$$

である、

ここで,

$$
\begin{aligned}
\boldsymbol{y}_{i, t} & =\left[y_{i, t-1}, \cdots, y_{i, t-n_{i}}\right]^{T} \\
\boldsymbol{u}_{t} & =\left[\boldsymbol{u}_{1, t}^{T}, \cdots, \boldsymbol{u}_{m, t}^{T}\right]^{T} \\
\boldsymbol{u}_{j, t} & =\left[u_{j, t-1}, \cdots, u_{j, t-n_{i}}\right]^{T} \\
\Delta \boldsymbol{y}_{i, t} & =\left[\Delta y_{i, t-1}, \cdots, \Delta y_{i, t-n_{i}}\right]^{T} \\
\Delta \boldsymbol{u}_{t} & =\left[\Delta \boldsymbol{u}_{1, t}^{T}, \cdots, \Delta \boldsymbol{u}_{m, t}^{T}\right]^{T} \\
\Delta \boldsymbol{u}_{j, t} & =\left[\Delta u_{j, t-1}, \cdots, \Delta u_{j, t-n_{i}}\right]^{T}
\end{aligned}
$$

とすると, (42) 式, (43) 式より,

$$
\left[\begin{array}{r}
y_{i, t} \\
\boldsymbol{y}_{i, t} \\
-\boldsymbol{u}_{t}
\end{array}\right]=\left[\begin{array}{r}
\eta_{i, t} \\
\boldsymbol{\eta}_{i, t} \\
-\boldsymbol{\xi}_{i, t}
\end{array}\right]+\left[\begin{array}{r}
\Delta y_{i, t} \\
\Delta \boldsymbol{y}_{i, t} \\
\Delta \boldsymbol{u}_{t}
\end{array}\right]
$$

となる。

さらに,

$$
\begin{aligned}
\boldsymbol{q}_{i, t}^{\prime} & =\left[y_{i, t}, \boldsymbol{y}_{i, t}^{T},-\boldsymbol{u}_{t}^{T}\right]^{T} \\
\Delta \boldsymbol{q}_{i, t} & =\left[\Delta y_{i, t}, \Delta \boldsymbol{y}_{i, t}^{T}, \Delta \boldsymbol{u}_{t}^{T}\right]^{T}
\end{aligned}
$$

とすると，

$$
\boldsymbol{q}_{i, t}^{\prime}=\boldsymbol{q}_{i, t}+\Delta \boldsymbol{q}_{i, t}
$$

となる。

また，雑音についての仮定より，

$$
E\left[\Delta \boldsymbol{q}_{i, t}\right]=0
$$

$$
\begin{aligned}
& E\left[\Delta \boldsymbol{q}_{i, t} \Delta \boldsymbol{q}_{i, t}^{T}\right] \\
& =\left[\begin{array}{cccc}
\hat{\sigma}_{y_{i}}^{2} I_{n_{i}+1} & 0 & \ldots & 0 \\
0 & \hat{\sigma}_{u_{1}}^{2} I_{n_{i}} & & 0 \\
\vdots & & \ddots & \\
0 & 0 & & \hat{\sigma}_{u_{m}}^{2} I_{n_{i}}
\end{array}\right]
\end{aligned}
$$

となる。なお, $E[*]$ は $t$ についての平均を表すものと する。

(37) 式より， $\boldsymbol{q}_{i, t}$ は $\boldsymbol{p}_{i}$ と直交する超平面に含まれる. しかし, 雑音 $\Delta \boldsymbol{q}_{i, t}$ によって, $\boldsymbol{q}_{i, t}^{\prime}$ は，その超平面外に あると考えられる。 そこで, 超平面から $\boldsymbol{q}_{i, t}^{\prime}$ までの距離 $e_{i, t}$ を考え, $e_{i, t}^{2}$ の総和を評価関数として,

$$
J=\sum_{t=1}^{N} e_{i, t}^{2}
$$

と定義し， $J$ を最小とする $\boldsymbol{p}_{i}$ をその推定值 $\hat{\boldsymbol{p}}_{i}$ とする.

まず, 距離 $e_{i, t}$ については, $\Delta y_{i, t}, \Delta u_{j, t}$ それぞれの 標準偏差の違いを考慮し, 誤差を標準偏差で割り正規化 したものを用いることとする。したがって， $\boldsymbol{p}_{i}$ と直交す る超平面上の任意のベクトル $\boldsymbol{q}$ を用いて, 次のように定 義できる。

$$
e_{i, t}^{2}=\min _{\boldsymbol{q}}\left\|W_{i}^{-1}\left(\boldsymbol{q}_{i, t}^{\prime}-\boldsymbol{q}\right)\right\|^{2}
$$

このとき, $W_{i}$ は $\hat{\sigma}_{u_{j}}, \hat{\sigma}_{y_{i}}$ によって, 次式で与えられる.

$$
W_{i}=\left[\begin{array}{cccc}
\hat{\sigma}_{y_{i}} I_{n_{i}+1} & 0 & \cdots & 0 \\
0 & \hat{\sigma}_{u_{1}} I_{n_{i}} & & 0 \\
\vdots & & \ddots & \\
0 & 0 & & \hat{\sigma}_{u_{m}} I_{n_{i}}
\end{array}\right]
$$

さて, $e_{i, t}^{2}$ を求めるため $\boldsymbol{q}$ を変数として，(37) 式，(57) 式より評価関数を, 


$$
\begin{aligned}
J_{1} & =\left(\boldsymbol{q}_{i, t}^{\prime}-\boldsymbol{q}\right)^{T} W_{i}^{-2}\left(\boldsymbol{q}_{i, t}^{\prime}-\boldsymbol{q}\right)+\lambda \boldsymbol{q}^{T} \hat{\boldsymbol{p}}_{i} \\
& =\boldsymbol{q}_{i, t}^{T} W_{i}^{-2} \boldsymbol{q}_{i, t}^{\prime}-2 \boldsymbol{q}^{T} W_{i}^{-2} \boldsymbol{q}_{i, t}^{\prime}+\boldsymbol{q}^{T} W_{i}^{-2} \boldsymbol{q} \\
& +\lambda \boldsymbol{q}^{T} \hat{\boldsymbol{p}}_{i}
\end{aligned}
$$

とする，そしてラグランジュの未定定数法を用いて， $J_{1}$ 最小の条件を求める。つまり, (59) 式を $\boldsymbol{q}, \lambda$ について偏 微分し 0 と㧍き， $\boldsymbol{q}$ を消去して $\lambda$ を求めることにより，

$$
\boldsymbol{q}_{i, t}^{\prime}-\boldsymbol{q}=\frac{\boldsymbol{q}_{i, t}^{\prime T} \hat{\boldsymbol{p}}_{i}}{\hat{\boldsymbol{p}}_{i}^{T} W_{i}^{2} \hat{\boldsymbol{p}}_{i}} W_{i}^{2} \hat{\boldsymbol{p}}_{i}
$$

が得られる。したがって，(57)式より，

$$
e_{i, t}^{2}=\frac{\left(\boldsymbol{q}_{i, t}^{\prime T} \hat{\boldsymbol{p}}_{i}\right)^{2}}{\hat{\boldsymbol{p}}_{i}^{T} W_{i}^{2} \hat{\boldsymbol{p}}_{i}}=\left(\boldsymbol{q}_{i, t}^{\prime T} W_{i}^{-1} \frac{W_{i} \hat{\boldsymbol{p}}_{i}}{\left\|W_{i} \hat{\boldsymbol{p}}_{i}\right\|}\right)^{2}
$$

となる．

$$
\text { ここで, }
$$

$$
\begin{aligned}
Z_{N} & =\left[\boldsymbol{q}_{i, 1}^{\prime}, \ldots, \boldsymbol{q}_{i, N}^{\prime}\right]^{T} W_{i}^{-1} \in R^{N \times n_{m i}} \\
\boldsymbol{n} & =\frac{W_{i} \hat{\boldsymbol{p}}_{i}}{\left\|W_{i} \hat{\boldsymbol{p}}_{i}\right\|} \in R^{n_{m i}}
\end{aligned}
$$

とおくと，(56)式，(61)式より，

$$
J=\left\|Z_{N} \boldsymbol{n}\right\|^{2}
$$

となる.

さらに, Jが最小となるときの $\boldsymbol{n}$ を求めることにより $\hat{\boldsymbol{p}}_{i}$ を決定する。評価関数を,

$$
J_{2}=\left(Z_{N} \boldsymbol{n}\right)^{T}\left(Z_{N} \boldsymbol{n}\right)+\lambda\left(1-\boldsymbol{n}^{T} \boldsymbol{n}\right)
$$

とする，そしてラグランジュの未定定数法を用いて， $\boldsymbol{n}, \lambda$ について偏微分し 0 とおくことにより，

$$
Z_{N}^{T} Z_{N} \boldsymbol{n}=\lambda \boldsymbol{n}
$$

となる。

したがって, (63)式, (66) 式の $\boldsymbol{n}$ は, $Z_{N}^{T} Z_{N}$ の単位 固有ベクトルとなる。 なお， $J$ の最小值は $Z_{N}^{T} Z_{N}$ の最小 固有值で与えられる。またそのとき， $\boldsymbol{n}$ を用いて，(63) 式より，

$$
\hat{\boldsymbol{p}}_{i}=\left\|W_{i} \hat{\boldsymbol{p}}_{i}\right\| W_{i}^{-1} \boldsymbol{n}
$$

となる。ここで,

$$
W_{i}^{-1} \boldsymbol{n}=\left[w_{1}, w_{2}, \cdots, w_{n_{m i}}\right]^{T}
$$

とすると，

$$
\hat{\boldsymbol{p}}_{i}=\left[1, \hat{\boldsymbol{\alpha}}_{i}^{T}, \hat{\boldsymbol{\beta}}_{i}^{T}\right]^{T}=\left\|W_{i} \hat{\boldsymbol{p}}_{i}\right\|\left[w_{1}, \ldots, w_{n_{m i}}\right]^{T}
$$

となり，

$$
\left\|W_{i} \hat{\boldsymbol{p}}_{i}\right\| w_{1}=1
$$

となる.よって (69)式，(70)式より，

$$
\left[\hat{\boldsymbol{\alpha}}_{i}^{T}, \hat{\boldsymbol{\beta}}_{i}^{T}\right]=w_{1}^{-1}\left[w_{2}, \ldots, w_{n_{m i}}\right]
$$

となり，

$$
\hat{\boldsymbol{p}}_{i}=w_{1}^{-1}\left[w_{1}, \cdots, w_{n_{m i}}\right]^{T}
$$

となる.

以上より，計測データ $\boldsymbol{q}_{i, t}^{\prime}$ と， $W_{i}^{-1}$ を用いて，(62) 式から $Z_{N}$ を作り, $Z_{N}^{T} Z_{N}$ の最小固有值に対する単位固 有べクトル $\boldsymbol{n}$ を求めると, $W_{i}^{-1}$ を用いて, (68) 式から $w_{1}, w_{2} \cdots w_{n_{m i}}$ が求められるので，(71) 式より $\hat{\boldsymbol{\alpha}}_{i}$ およ び $\hat{\boldsymbol{\beta}}_{i}$ すなわち $\hat{\boldsymbol{\beta}}_{i j}$ が求められ，(72)式より, $\hat{\boldsymbol{p}}_{i}$ が求め られることが示された。

ところで，重み行列 $W_{i}$ の要素は， $\hat{\sigma}_{y_{i}}, \hat{\sigma}_{u_{k}}$ であるた め，それらの中に 0 に近くなるものがあると， $W_{i}^{-1}$ が 計算できなくなる可能性がある.

$W_{i}^{-1}$ は, (58) 式より，

$$
W_{i}^{-1}=\left[\begin{array}{cccc}
\hat{\sigma}_{y_{i}}^{-1} I_{n_{i}+1} & 0 & \cdots & 0 \\
0 & \hat{\sigma}_{u_{1}}^{-1} I_{n_{i}} & & 0 \\
\vdots & & \ddots & \\
0 & 0 & & \hat{\sigma}_{u_{m}}^{-1} I_{n_{i}}
\end{array}\right]
$$

となる.ここで, $j=1, \ldots, m$ として

$$
\begin{aligned}
\sigma_{A} & =\hat{\sigma}_{y_{i}} \hat{\sigma}_{u_{1}} \cdots \hat{\sigma}_{u_{m}} \\
\sigma_{Y_{i}} & =\hat{\sigma}_{u_{1}} \cdots \hat{\sigma}_{u_{m}} \\
\sigma_{U_{i j}} & =\hat{\sigma}_{y_{i}} \hat{\sigma}_{u_{j}}^{-1} \prod_{k=1}^{m} \hat{\sigma}_{u_{k}}=\hat{\sigma}_{y_{i}} \prod_{k \neq j} \hat{\sigma}_{u_{k}}
\end{aligned}
$$

とする。 そして, $W_{i}^{\prime-1}$ を，

$$
\begin{aligned}
W_{i}^{\prime-1} & =\sigma_{A} W_{i}^{-1} \\
& =\left[\begin{array}{cccc}
\sigma_{Y_{i}} I_{n_{i}+1} & 0 & \cdots & 0 \\
0 & \sigma_{U_{i 1}} I_{n_{i}} & & 0 \\
\vdots & & \ddots & \\
0 & 0 & & \sigma_{U_{i m}} I_{n_{i}}
\end{array}\right]
\end{aligned}
$$

と定義する.

ここで, (57) 式に扔いて導入された $W_{i}$ は $\Delta \boldsymbol{q}_{i, t}$ の要 素の標準偏差の比を考慮したものであり， $\sigma_{A}$ 倍してもよ い. そこで, $W_{i}^{\prime-1}$ を(57) 式の $W_{i}^{-1}$ の代わりに使うこ とを提案する。これにより，(76) 式の $\hat{\sigma}_{u_{j}}^{-1}$ が消去され， $\hat{\sigma}_{y_{i}}, \hat{\sigma}_{u_{k}}$ の中に 0 に近くなるものがあっても, $W_{i}^{-1}$ が計 算可能となる.

なお， $\hat{\sigma}_{y_{i}}, \hat{\sigma}_{u_{k}}$ は， $\sigma_{y_{i}}, \sigma_{u_{k}}$ の計算上の推定值であり， $\sigma_{y_{i}}, \sigma_{u_{k}}$ が 0 に近くなくても, デー夕数が少ない場合な どでは，雑音の影響により值が変動し 0 に近くなること がある，そのような場合， $W_{i}$ の要素には発散する要素 が現れ計算不能となる。 そこで，すでに発表された論文 $[1,2]$ による方法では, $\hat{\sigma}_{y_{i}}, \hat{\sigma}_{u_{k}}$ の下限值を設けることで それを回避していた，ただし下限值以下の值は求めるこ 
とができないため, 下限值は可能な限り小さな值にする 必要があるが，計算能力による限界がある。とくに標準 偏差の真值が 0 に近い場合，そのような下限值を選ぶこ とが困難となる。

しかし， $\hat{\boldsymbol{p}}_{i}$ は $W_{i}$ の逆行列を用いて計算されるので, $W_{i}^{\prime-1}$ を使うという本提案方法では，そのような下限值 を設ける必要がなくなり，より有効な計算方法となって いる.

また，

$$
Q_{N}=\left[\boldsymbol{q}_{i, 1}^{\prime}, \ldots, \boldsymbol{q}_{i, N}^{\prime}\right]
$$

とすると (62) 式より，

$$
\begin{aligned}
Z_{N+1}^{T} Z_{N+1} & =\left(W_{i}^{-1}\right)^{T} Q_{N+1} Q_{N+1}^{T} W_{i}^{-1} \\
Q_{N+1} Q_{N+1}^{T} & =Q_{N} Q_{N}^{T}+\boldsymbol{q}_{i, N+1}^{\prime} \boldsymbol{q}_{i, N+1}^{T}
\end{aligned}
$$

となり， $N$ に対して $Z_{N}^{T} Z_{N}$ を逐次的に求めることがで き，計算の高速化が可能である。

\section{$2.4 \hat{\boldsymbol{p}}_{i}$ を用いて $\hat{\sigma}_{u_{j}}^{2}, \hat{\sigma}_{y_{i}}^{2}$ を求める方法}

同定アルゴリズムの S2 における， $\hat{\boldsymbol{p}}_{i}$ を用いて $\hat{\sigma}_{u_{j}}^{2}, \hat{\sigma}_{y_{i}}^{2}$ を求める方法について述べる.

なお本節では，推定されたパラメータベクトルを $\boldsymbol{\alpha}_{i}$, $\boldsymbol{\beta}_{i}$ のように表し，`記号を省略する。

まず, $k, \ell\left(1 \leq k<\ell \leq n_{i}\right)$ および, $i, j(1 \leq i \leq r, 1 \leq j \leq$ $m)$ に対して, $\Delta k=\ell-k+1$ として， $\boldsymbol{\alpha}_{i, k: \ell}, \boldsymbol{\beta}_{i, k: \ell}$ を,

$$
\begin{aligned}
\boldsymbol{\alpha}_{i, k: \ell} & =\left[\alpha_{i, k}, \alpha_{i, k+1}, \cdots, \alpha_{i, \ell}\right]^{T} \in R^{\Delta k} \\
\boldsymbol{\beta}_{i, k: \ell} & =\left[\boldsymbol{\beta}_{i 1, k: \ell}^{T}, \cdots, \boldsymbol{\beta}_{i m, k: \ell}^{T}\right]^{T} \in R^{m \Delta k} \\
\boldsymbol{\beta}_{i j, k: \ell} & =\left[\beta_{i j, k}, \beta_{i j, k+1}, \cdots, \beta_{i j, \ell}\right]^{T} \in R^{\Delta k}
\end{aligned}
$$

とする.さらに, $k, \ell(1 \leq k<\ell \leq N)$ に対して,$\quad \boldsymbol{\eta}_{i, \ell: k}$, $\boldsymbol{\xi}_{i, \ell: k}$ を,

$$
\begin{aligned}
\boldsymbol{\eta}_{i, \ell: k} & =\left[\eta_{i, \ell}, \eta_{i, \ell-1}, \cdots, \eta_{i, k}\right]^{T} \in R^{\Delta k} \\
\boldsymbol{\xi}_{\ell: k} & =\left[\boldsymbol{\xi}_{1, \ell: k}, \cdots, \boldsymbol{\xi}_{m, \ell: k}\right]^{T} \in R^{m \Delta k} \\
\boldsymbol{\xi}_{j, \ell: k} & =\left[\xi_{j, \ell}, \xi_{j, \ell-1}, \cdots, \xi_{j, k}\right]^{T} \in R^{\Delta k}
\end{aligned}
$$

とする。

まず, (26) 式より，

$$
\eta_{i, t}=-\boldsymbol{\alpha}_{i, 1: n_{i}}^{T} \boldsymbol{\eta}_{i, t-1: t-n_{i}}+\boldsymbol{\beta}_{i, 1: n_{i}}^{T} \boldsymbol{\xi}_{i, t-1: t-n_{i}}
$$

が得られる。また，(87)式の $t を t-1$ とすることにより，

$$
\eta_{i, t-1}=-\boldsymbol{\alpha}_{i, 1: n_{i}}^{T} \boldsymbol{\eta}_{i, t-2: t-n_{i}-1}+\boldsymbol{\beta}_{i, 1: n_{i}}^{T} \boldsymbol{\xi}_{t-2: t-n_{i}-1}
$$

となる。この (88) 式を用いると，(87) 式は,

$$
\begin{aligned}
\eta_{i, t}= & -\alpha_{i, 1} \eta_{i, t-1}-\boldsymbol{\alpha}_{i, 2: n_{i}}^{T} \boldsymbol{\eta}_{i, t-2: t-n_{i}} \\
& +\boldsymbol{\beta}_{i, 1: n_{i}}^{T} \boldsymbol{\xi}_{t-1: t-n_{i}} \\
= & -\alpha_{i, 1}\left(-\boldsymbol{\alpha}_{i, 1: n_{i}}^{T} \boldsymbol{\eta}_{i, t-2: t-n_{i}-1}\right. \\
& \left.+\boldsymbol{\beta}_{i, 1: n_{i}}^{T} \boldsymbol{\xi}_{t-2: t-n_{i}-1}\right)
\end{aligned}
$$

$$
\begin{aligned}
& -\boldsymbol{\alpha}_{i, 2: n_{i}}^{T} \boldsymbol{\eta}_{i, t-2: t-n_{i}}+\boldsymbol{\beta}_{i, 1: n_{i}}^{T} \boldsymbol{\xi}_{t-1: t-n_{i}} \\
= & \left(\alpha_{i, 1} \boldsymbol{\alpha}_{i, 1: n_{i}}^{T}-\left[\boldsymbol{\alpha}_{i, 2: n_{i}}^{T}, 0\right]\right) \boldsymbol{\eta}_{i, t-2: t-n_{i}-1} \\
& +\left(-\alpha_{i, 1}\left[0, \boldsymbol{\beta}_{i, 1: n_{i}}^{T}\right]+\left[\boldsymbol{\beta}_{i, 1: n_{i}}^{T}, 0\right]\right) \boldsymbol{\xi}_{t-1: t-n_{i}-1}
\end{aligned}
$$

となり，これより，

$$
\eta_{i, t}=\boldsymbol{c}_{2, i}^{T} \boldsymbol{\eta}_{i, t-2: t-n_{i}-1}+\boldsymbol{d}_{2, i}^{T} \boldsymbol{\xi}_{t-1: t-n_{i}-1}
$$

ただし

$$
\begin{aligned}
\boldsymbol{c}_{2, i} & =\alpha_{i, 1} \boldsymbol{\alpha}_{i, 1: n_{i}}-\left[\boldsymbol{\alpha}_{i, 2: n_{i}}^{T}, 0\right]^{T} \in R^{n_{i}} \\
\boldsymbol{d}_{2, i} & =\left[\boldsymbol{d}_{2, i, 1}^{T}, \boldsymbol{d}_{2, i, 2}^{T}, \ldots, \boldsymbol{d}_{2, i, m}^{T}\right]^{T} \in R^{m\left(n_{i}+1\right)} \\
\boldsymbol{d}_{2, i, j} & =-\alpha_{i, 1}\left[0, \boldsymbol{\beta}_{i j, 1: n_{i}}^{T}\right]^{T}+\left[\boldsymbol{\beta}_{i j, 1: n_{i}}^{T}, 0\right]^{T} \in R^{n_{i}+1}
\end{aligned}
$$

となる。また， $h=1, \ldots, h_{0}$ に対して，

$$
t_{h}=t-h
$$

とし,

$$
n_{I}=n_{i}-1
$$

とすると，

$$
\eta_{i, t}=\boldsymbol{c}_{2, i}^{T} \boldsymbol{\eta}_{i, t_{2}: t_{2}-n_{I}}+\boldsymbol{d}_{2, i}^{T} \boldsymbol{\xi}_{t_{1}: t_{2}-n_{I}}
$$

となる

また, $h=1$ の場合については $(87)$ 式より,

$$
\begin{aligned}
\eta_{i, t} & =\boldsymbol{c}_{1, i}^{T} \boldsymbol{\eta}_{i, t_{1}: t_{1}-n_{I}}+\boldsymbol{d}_{1, i}^{T} \boldsymbol{\xi}_{t_{1}: t_{1}-n_{I}} \\
\boldsymbol{c}_{1, i} & =-\boldsymbol{\alpha}_{i, 1: n_{I}+1} \\
\boldsymbol{d}_{1, i} & =\boldsymbol{\beta}_{i, 1: n_{I}+1}
\end{aligned}
$$

となる。

さらに，代入計算によって $h=2, \cdots, h_{0}$ に対して $\boldsymbol{c}_{h, i}$, $\boldsymbol{d}_{h, i}$ が求められ, それらを使って,

$$
\eta_{i, t}=\boldsymbol{c}_{h, i}^{T} \boldsymbol{\eta}_{i, t_{h}: t_{h}-n_{I}}+\boldsymbol{d}_{h, i}^{T} \boldsymbol{\xi}_{t_{1}: t_{h}-n_{I}}
$$

と表すことができる

なお，

$$
\begin{aligned}
\boldsymbol{c}_{h, i} & =\left[c_{h, i, 1}, \cdots, c_{h, i, n_{I}+1}\right]^{T} \in R^{n_{I}+1} \\
\boldsymbol{d}_{h, i} & =\left[\boldsymbol{d}_{h, i, 1}^{T}, \cdots, \boldsymbol{d}_{h, i, m}^{T}\right]^{T} \in R^{m\left(n_{I}+h\right)} \\
\boldsymbol{d}_{h, i, j} & =\left[d_{h, i, j, 1}, \cdots, d_{h, i, j, n_{I}+h}\right]^{T} \in R^{n_{I}+h}
\end{aligned}
$$

とする。

ここで, $k, \ell(1 \leq k<\ell \leq N)$ に対して,$\Delta k=\ell-k+1$ として, $\boldsymbol{y}_{i, \ell: k}, \boldsymbol{u}_{\ell: k}, \Delta \boldsymbol{y}_{i, \ell: k}, \Delta \boldsymbol{u}_{\ell: k}$ を,

$$
\begin{aligned}
\boldsymbol{y}_{i, \ell: k} & =\left[y_{i, \ell}, y_{i, \ell-1}, \cdots, y_{i, k}\right]^{T} \in R^{\Delta k} \\
\boldsymbol{u}_{\ell: k} & =\left[\boldsymbol{u}_{1, \ell: k}, \cdots, \boldsymbol{u}_{m, \ell: k}\right]^{T} \in R^{m \Delta k} \\
\boldsymbol{u}_{j, \ell: k} & =\left[u_{j, \ell}, u_{j, \ell-1}, \cdots, u_{j, k}\right]^{T} \in R^{\Delta k} \\
\Delta \boldsymbol{y}_{i, \ell: k} & =\left[\Delta y_{i, \ell}, \Delta y_{i, \ell-1}, \cdots, \Delta y_{i, k}\right]^{T} \in R^{\Delta k}
\end{aligned}
$$




$$
\begin{aligned}
\Delta \boldsymbol{u}_{\ell: k} & =\left[\Delta \boldsymbol{u}_{1, \ell: k}, \cdots, \Delta \boldsymbol{u}_{m, \ell: k}\right]^{T} \in R^{m \Delta k} \\
\Delta \boldsymbol{u}_{j, \ell: k} & =\left[\Delta u_{j, \ell}, \Delta u_{j, \ell-1}, \cdots, \Delta u_{j, k}\right]^{T} \in R^{\Delta k}
\end{aligned}
$$

とすると，(42)式，(43)式より，(102)式は,

$$
\begin{aligned}
y_{i, t}-\Delta y_{i, t} & =\boldsymbol{c}_{h, i}^{T}\left(\boldsymbol{y}_{i, t_{h}: t_{h}-n_{I}}-\Delta \boldsymbol{y}_{i, t_{h}: t_{h}-n_{I}}\right) \\
& +\boldsymbol{d}_{h, i}^{T}\left(\boldsymbol{u}_{t_{1}: t_{h}-n_{I}}+\Delta \boldsymbol{u}_{t_{1}: t_{h}-n_{I}}\right)
\end{aligned}
$$

となる。したがって，

$$
\begin{aligned}
& \Delta y_{i, t}-\boldsymbol{c}_{h, i}^{T} \Delta \boldsymbol{y}_{i, t_{h}: t_{h}-n_{I}}+\boldsymbol{d}_{h, i}^{T} \Delta \boldsymbol{u}_{t_{1}: t_{h}-n_{I}} \\
& =y_{i, t}-\boldsymbol{c}_{h, i}^{T} \boldsymbol{y}_{i, t_{h}: t_{h}-n_{I}}-\boldsymbol{d}_{h, i}^{T} \boldsymbol{u}_{t_{1}: t_{h}-n_{I}}
\end{aligned}
$$

となる。ここで,

$$
\epsilon_{h, i, t}=y_{i, t}-\boldsymbol{c}_{h, i}^{T} \boldsymbol{y}_{i, t_{h}: t_{h}-n_{I}}-\boldsymbol{d}_{h, i}^{T} \boldsymbol{u}_{t_{1}: t_{h}-n_{I}}
$$

とすると, (113) 式は,

$$
\Delta y_{i, t}-\boldsymbol{c}_{h, i}^{T} \Delta \boldsymbol{y}_{i, t_{h}: t_{h}-n_{I}}+\boldsymbol{d}_{h, i}^{T} \Delta \boldsymbol{u}_{t_{1}: t_{h}-n_{I}}=\epsilon_{h, i, t}
$$

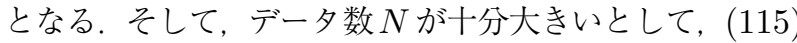
式の両辺の二乗平均をとると,

$$
\left(1+\left\|\boldsymbol{c}_{h, i}\right\|^{2}\right) \sigma_{y_{i}}^{2}+\sum_{j=1}^{m}\left\|\boldsymbol{d}_{h, i, j}\right\|^{2} \sigma_{u_{j}}^{2}=E\left[\epsilon_{h, i, t}^{2}\right]
$$

となる．

よって (116) 式は， $\sigma_{u_{j}}^{2}, \sigma_{y_{i}}^{2}$ についての線形な方程式 となる．

ここで, $h_{0}>\frac{m}{r}+1$ として,

$$
\begin{aligned}
& F=\left[\begin{array}{cc}
F_{C, 1} & F_{D, 1} \\
\vdots & \vdots \\
F_{C, h_{0}} & F_{D, h_{0}}
\end{array}\right] \in R^{h_{0} r \times(r+m)} \\
& F_{C, h}=\left[\begin{array}{ccc}
1+\left\|c_{h, 1}\right\|^{2} & & 0 \\
& \ddots & \\
0 & & 1+\left\|\boldsymbol{c}_{h, r}\right\|^{2}
\end{array}\right] \\
& F_{D, h}=\left[\begin{array}{ccc}
\left\|\boldsymbol{d}_{h, 11}\right\|^{2} & \cdots & \left\|\boldsymbol{d}_{h, 1 m}\right\|^{2} \\
\vdots & \\
\left\|\boldsymbol{d}_{h, r 1}\right\|^{2} & \cdots & \left\|\boldsymbol{d}_{h, r m}\right\|^{2}
\end{array}\right]
\end{aligned}
$$

また，

$$
\begin{aligned}
\phi & =\left[\hat{\sigma}_{y_{1}}^{2}, \ldots, \hat{\sigma}_{y_{r}}^{2}, \hat{\sigma}_{u_{1}}^{2}, \ldots, \hat{\sigma}_{u_{m}}^{2}\right]^{T} \in R^{r+m} \\
\boldsymbol{\psi} & =\left[\boldsymbol{\psi}_{1}, \ldots, \boldsymbol{\psi}_{h_{0}}\right]^{T} \in R^{h_{0} r} \\
\boldsymbol{\psi}_{h} & =\left[E\left[\epsilon_{h, 1, t}^{2}\right], \ldots, E\left[\epsilon_{h, r, t}^{2}\right]\right]^{T}
\end{aligned}
$$

とすると，次式が得られる.

$$
F \phi=\psi
$$

したがって，最小二乗法により $\hat{\sigma}_{u_{j}}^{2}, \hat{\sigma}_{y_{i}}^{2}$ を要素とする ベクトル $\phi$ は次式で求められる.

$$
\phi=\left(F^{T} F\right)^{-1} F^{T} \boldsymbol{\psi}
$$

以上より， $\boldsymbol{c}_{h, i}, \boldsymbol{d}_{h, i}$ から (117) 式，(118) 式，(119) 式 を用いて $F$ を作り，計測データ $\boldsymbol{y}_{i, t} \boldsymbol{u}_{j}$ から (114) 式を用 いて $\epsilon_{h, i, t}$ を求め, (120) 式，(121) 式を用いて $\psi$ を作る ことにより，(123)式を用いて $\phi$ が求められ， $\hat{\sigma}_{y_{1}}^{2}, \ldots, \hat{\sigma}_{y_{r}}^{2}$ および， $\hat{\sigma}_{u_{1}}^{2}, \ldots, \hat{\sigma}_{u_{m}}^{2}$ が同時に求められることが示さ れた。

ところで, $\boldsymbol{c}_{h, i}, \boldsymbol{d}_{h, i}$ は，(87) 式から (95) 式までと同 様に代入計算を繰り返すことによって導出される，ただ し，つぎに述べる計算プログラムでその值を求めること が可能である.

\section{$2.5 c_{h, i}, d_{h, i}$ の計算プログラム}

ここでは, 前節の $\boldsymbol{c}_{h, i}, \boldsymbol{d}_{h, i}$ の要素 $c_{h, i, k}, d_{h, i, j, k}$ を再 帰関数によって求めるアルゴリズムについて述べる.

ただし，MISO に対する同定法 [2] と共通であるので， 詳細は省略する。

まず，次のように (99) 式の右辺を関数 $f_{i, 1}$ としてプロ グラム中で定義する。

$$
\begin{aligned}
f_{i, 1}\left(\boldsymbol{U}^{(1)}, \boldsymbol{Y}\right) & :=\boldsymbol{c}_{1, i}^{T} \boldsymbol{Y}+\boldsymbol{d}_{1, i}^{T} \boldsymbol{U}^{(1)} \\
\boldsymbol{Y} & =\boldsymbol{\eta}_{i, t_{1}: t_{1}-n_{I}} \in \mathbf{R}^{n_{I}+1} \\
\boldsymbol{U}^{(1)} & =\boldsymbol{\xi}_{t_{1}: t_{1}-n_{I}} \in \mathbf{R}^{m\left(n_{I}+1\right)}
\end{aligned}
$$

同様に (102) 式の右辺は, 次のような関数 $f_{i, h}$ として プログラム中で定義する。

$$
\begin{aligned}
f_{i, h}\left(\boldsymbol{U}^{(h)}, \boldsymbol{Y}\right) & =\boldsymbol{c}_{h, i}^{T} \boldsymbol{Y}+\boldsymbol{d}_{h, i}^{T} \boldsymbol{U}^{(h)} \\
\boldsymbol{Y} & =\boldsymbol{\eta}_{i, t_{h}: t_{h}-n_{I}} \in \mathbf{R}^{n_{I}+1} \\
\boldsymbol{U}^{(h)} & =\boldsymbol{\xi}_{t_{1}: t_{h}-n_{I}} \in \mathbf{R}^{m\left(n_{I}+h\right)}
\end{aligned}
$$

そして,

$$
\begin{aligned}
\boldsymbol{Y} & =\left[Y_{1}, \cdots, Y_{n_{I}+1}\right]^{T} \in \mathbf{R}^{n_{I}+1} \\
\boldsymbol{U}^{(h)} & =\left[\boldsymbol{U}_{1}^{T}, \cdots, \boldsymbol{U}_{j}^{T}, \cdots, \boldsymbol{U}_{m}^{T}\right]^{T} \in \mathbf{R}^{m\left(n_{I}+h\right)} \\
\boldsymbol{U}_{j} & =\left[U_{j, 1}^{T}, \cdots, U_{j, n_{I}+h}^{T}\right]^{T} \in \mathbf{R}^{n_{I}+h}
\end{aligned}
$$

とし, $\boldsymbol{U}_{k: \ell}^{(h)}, \boldsymbol{Y}_{k: \ell}(k<\ell)$ を

$$
\begin{aligned}
\boldsymbol{Y}_{k: \ell} & =\left[Y_{k}, \cdots, Y_{\ell}\right]^{T} \\
\boldsymbol{U}_{k: \ell}^{(h)} & =\left[\boldsymbol{U}_{1, k: \ell}^{T}, \cdots, \boldsymbol{U}_{j, k: \ell}^{T}, \cdots, \boldsymbol{U}_{m, k: \ell}^{T}\right]^{T} \\
\boldsymbol{U}_{j, k: \ell} & =\left[U_{j, k}, \cdots, U_{j, \ell}\right]^{T}
\end{aligned}
$$

とする.

このとき, $f_{i, h}\left(\boldsymbol{U}^{(h)}, \boldsymbol{Y}\right)$ は次の例のようにプログラ 厶中で再㷌的に定義することが可能である [2].

$\cdot f_{i, h}$ の計算プログラムにおける定義の例 $\cdot h=1$ : 
$\boldsymbol{c}_{1, i}^{T} \boldsymbol{Y}+\boldsymbol{d}_{1, i}^{T} \boldsymbol{U}^{(1)}$

- $h>1$ :

$f_{i, h-1}\left(\boldsymbol{U}_{1: n_{I}+h-1}^{(h)},\left[f_{i, 1}\left(\boldsymbol{U}_{h: n_{I}+h}^{(h)}, \boldsymbol{Y}_{1: n_{I}+1}\right), \boldsymbol{Y}_{1: n_{I}}^{T}\right]^{T}\right)$

まず, $c_{h, i, k}$ は, $k=1, \ldots, n_{I}+1$ に対して, 次式で与 えられる。

$$
\begin{aligned}
& c_{h, i, k}=f_{i, h}\left(\boldsymbol{U}^{(h)}, \boldsymbol{Y}\right) \\
& \boldsymbol{U}^{(h)}=0 \\
& \boldsymbol{Y}=\left[Y_{1}, \cdots, Y_{\ell}, \cdots, Y_{n_{I}+1}\right]^{T} \\
& \left(\ell=1, \ldots, n_{I}+1\right) \\
& Y_{\ell}= \begin{cases}1 & (\ell=k) \\
0 & (\ell \neq k)\end{cases}
\end{aligned}
$$

また, $d_{h, i, j, k}$ は, $k=1, \ldots, n_{I}+h$ に対して, 次式で与 えられる。

$$
\begin{aligned}
& d_{h, i, j, k}=f_{i, h}\left(\boldsymbol{U}^{(h)}, \boldsymbol{Y}\right) \\
& \boldsymbol{Y}=0 \\
& \boldsymbol{U}^{(h)}=\left[\boldsymbol{U}_{1}^{T}, \cdots, \boldsymbol{U}_{m}^{T}\right]^{T} \\
& \boldsymbol{U}_{j}=\left[U_{j, 1}, \cdots U_{j, \ell}, \cdots, U_{j, n_{I}+h}\right]^{T} \\
& \left(\ell=1, \ldots, m\left(n_{I}+h\right)\right) \\
& U_{j, \ell}= \begin{cases}1 & \left(\ell=k+(j-1)\left(n_{I}+h\right)\right) \\
0 & \left(\ell \neq k+(j-1)\left(n_{I}+h\right)\right)\end{cases}
\end{aligned}
$$

\section{3. ニ入カニ出カシステムに対するシミュ レーション}

システムおよび誤差を仮定し，パラメータ同定を行う シミュレーションを実施した。

システムは Fig. 1 において,

$$
\begin{aligned}
G_{11}(z) & =\frac{1.0 z^{-1}+0.5 z^{-2}}{1-0.8 \sqrt{3} z^{-1}+0.64 z^{-2}} \\
G_{12}(z) & =\frac{-1.4 z^{-1}-0.7 z^{-2}}{1-0.8 z^{-1}+0.64 z^{-2}} \\
G_{21}(z) & =\frac{1.0 z^{-1}+0.5 z^{-2}}{1+0.8 z^{-1}+0.64 z^{-2}}
\end{aligned}
$$

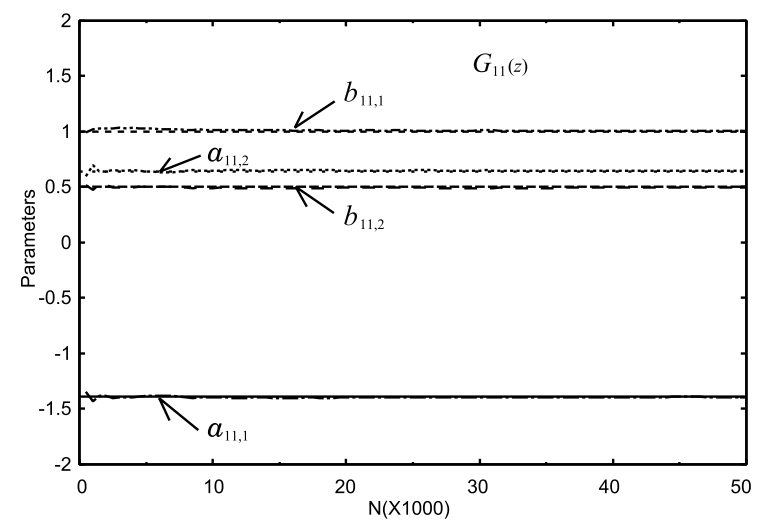

Fig. 2 Estimated parameters of $G_{11}(z)$

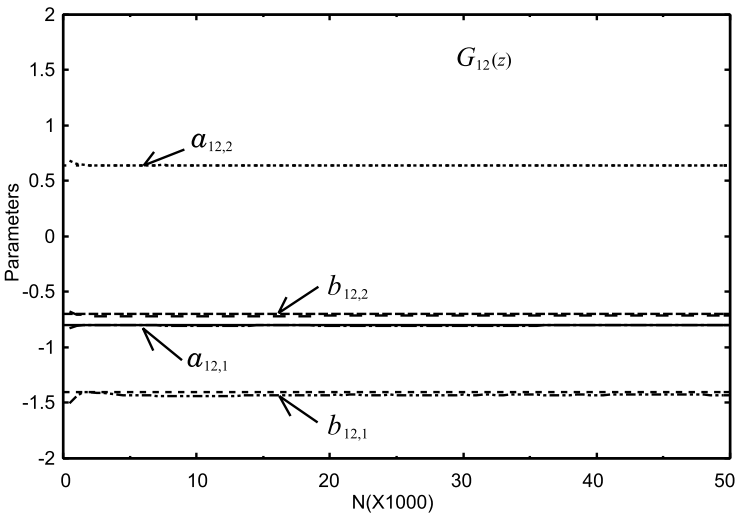

Fig. 3 Estimated parameters of $G_{12}(z)$

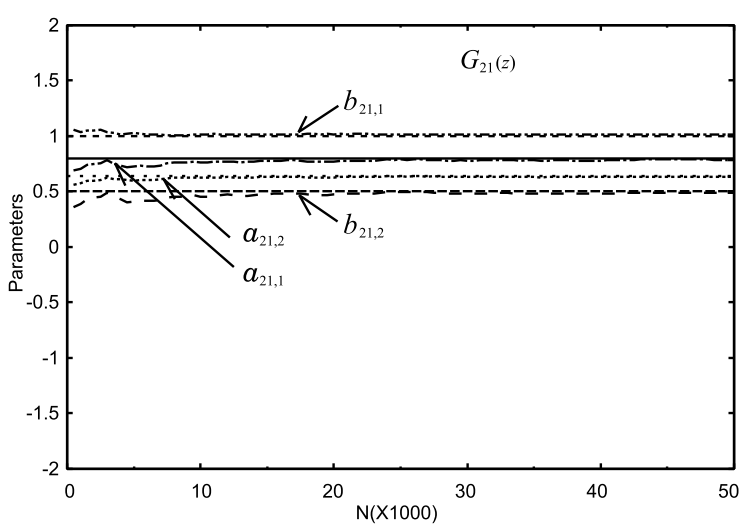

Fig. 4 Estimated parameters of $G_{21}(z)$

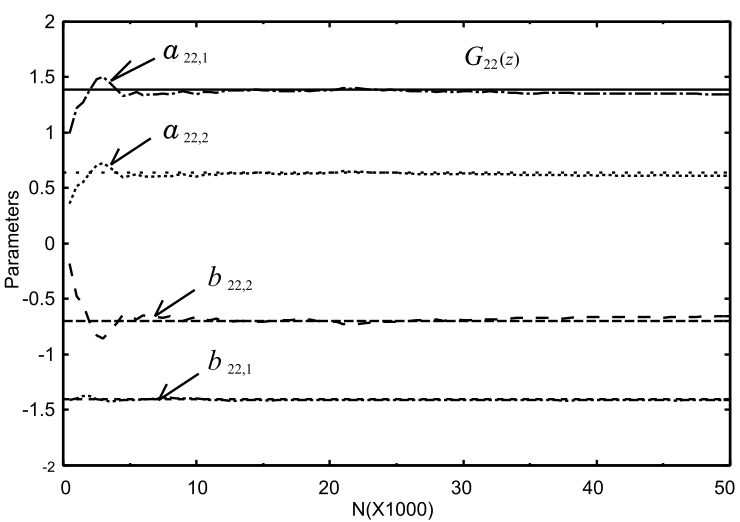

Fig. 5 Estimated parameters of $G_{22}(z)$

$$
G_{22}(z)=\frac{-1.4 z^{-1}-0.7 z^{-2}}{1+0.8 \sqrt{3} z^{-1}+0.64 z^{-2}}
$$

としたものであり $, r=2, \quad m=2, \quad n_{11}=n_{12}=n_{21}=$ $n_{22}=2$ となる．また，入力は \pm 1 の值をとるランダム 信号であり, 入力 $u$, 出力 $y$ の誤差 $\Delta u, \Delta y$ の分散を $\sigma_{u_{1}}^{2}=0.01, \sigma_{u_{2}}^{2}=0.02, \sigma_{y_{1}}^{2}=0.04, \sigma_{y_{2}}^{2}=0.08$ とした. また，同定計算に必要なパラメータについては， $h_{0}=3$, $\mu=0.0001$ とした.

入出力データをシミュレーション計算で発生させ, デー 夕数 500 ごとにその時点でのデータ全体（総数は増加す 


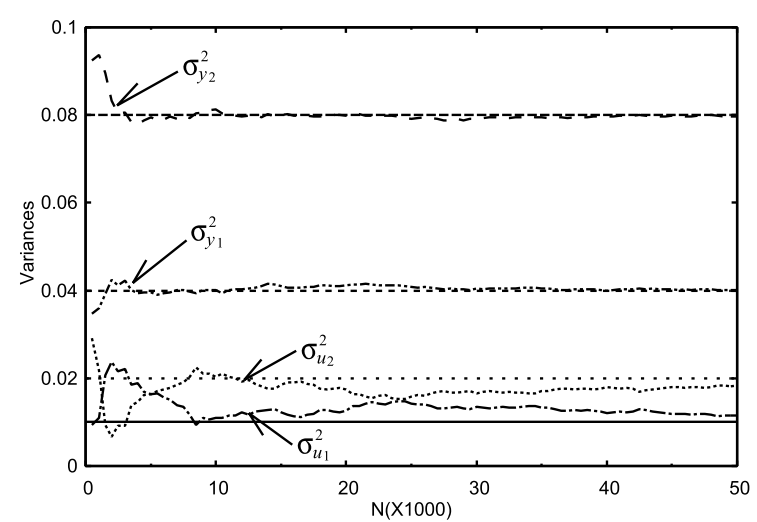

Fig. 6 Estimated variances

Table 1 Results with simulation

\begin{tabular}{|c||r|r|r|}
\hline $\boldsymbol{p}_{G k}, \sigma^{2}$ & T.V. & E.V. & E.V.T.V. \\
\hline \hline$a_{11,1}$ & -1.386 & -1.394 & -0.0086 \\
\hline$a_{11,2}$ & 0.640 & 0.648 & 0.0084 \\
\hline$b_{11,1}$ & 1.000 & 1.010 & 0.0097 \\
\hline$b_{11,2}$ & 0.500 & 0.495 & -0.0051 \\
\hline \hline$a_{12,1}$ & -0.800 & -0.802 & -0.0015 \\
\hline$a_{12,2}$ & 0.640 & 0.641 & 0.0012 \\
\hline$b_{12,1}$ & -1.400 & -1.429 & -0.0290 \\
\hline$b_{12,2}$ & -0.700 & -0.713 & -0.0127 \\
\hline \hline$a_{21,1}$ & 0.800 & 0.786 & -0.0140 \\
\hline$a_{21,2}$ & 0.640 & 0.634 & -0.0061 \\
\hline$b_{21,1}$ & 1.000 & 1.014 & 0.0145 \\
\hline$b_{21,2}$ & 0.500 & 0.486 & -0.0135 \\
\hline \hline$a_{22,1}$ & 1.386 & 1.344 & -0.0414 \\
\hline$a_{22,2}$ & 0.640 & 0.609 & -0.0307 \\
\hline$b_{22,1}$ & -1.400 & -1.413 & -0.0132 \\
\hline$b_{22,2}$ & -0.700 & -0.654 & 0.0463 \\
\hline \hline$\sigma_{u_{1}}^{2}$ & 0.01 & 0.0114 & 0.0014 \\
\hline$\sigma_{u_{2}}^{2}$ & 0.02 & 0.0184 & -0.0016 \\
\hline$\sigma_{y_{1}}^{2}$ & 0.04 & 0.0401 & 0.0001 \\
\hline$\sigma_{y_{2}}^{2}$ & 0.08 & 0.0796 & 0.0004 \\
\hline \hline
\end{tabular}

T.V.: True values, E.V.: Estimated values

る） $\boldsymbol{q}_{i, t}(t=1, \ldots, N)$ を用いて, パラメータ同定を行った. $N$ に対する伝達関数パラメー夕の推定值の変化を Fig. 2, Fig. 3, Fig. 4, Fig. 5 に示す. また, 誤差の分散 の変化を Fig. 6 に示す.

そして $N=50000$ において得られたパラメータおよび 誤差の分散の推定值を Table 1 に示す.

\section{4. 考察}

本同定法を用いたパラメータ同定のシミュレーション の結果について考察する.

まずFig. 2, Fig. 5 より，N>20000において各パラ
メータがほぼ正しく推定されていることがわかる.

また Fig. 6 より，分散の值は $N=50000$ で真值に 近づいていることがわかる。そのとき, Table 1 より， $\sigma_{u_{1}}^{2}, \sigma_{u_{2}}^{2}, \sigma_{y_{1}}^{2}, \sigma_{y_{1}}^{2}$ の残差は, $0.0014,-0.0016,0.0001$, 0.0004 程度であることがわかる.

なお，本同定法の繰り返し計算アルゴリズムにおける 収束性については, SISO システムの場合 [1] と同様に検 証可能である.

\section{5. 結論}

今回, MIMO システムの同定法を構成するため, すで に提案された入出力誤差分散推定を用いた SISO システ ムのパラメータ同定法および MISO システムのパラメー 夕同定を拡張し，そのアルゴリズムを提案した。 そして， 二入力二出力システムに対するシミュレーションを行い, パラメータ推定值が得られることを確認した。

本同定法は入力誤差を考慮しており, 入出力誤差の分 散が求められるので, システムの入出力誤差の分散の值 を必要とする場合，本方式の利用が可能であると考えら れる。

たとえば移動体制御システムでは，移動に伴い，動特 性, ノイズ特性が変化するため, それらの変化に応じて 制御方法を変化させることが有効となると考えられるが, そのようなシステムにおいて利用できる同定法といえる.

今後実験システムへ適用し, 本同定法の有効性につい て検証を行う必要があると考えられる。

\section{参 考文 献}

[1] 小松: 入出力誤差分散推定を用いた一入力一出力システ ムのパラメータ同定アルゴリズム; システム制御情報学 会論文誌, Vol. 27, No. 5, pp. 216-229 (2014)

[2] 小松: 入出力誤差分散推定を用いた多入力一出力システ ムのパラメータ同定法; システム制御情報学会論文誌, Vol. 28, No. 7, pp. 310-319 (2015)

[3] 中川, 小柳: 最小二乗法による実験データ解析一プロ グラムSALS (UP 応用数学選書 7), 東京大学出版会 (1982)

[4] 相良, 秋月, 中溝, 片山 : システム同定, (社) 計測自動 制御学会編, コロナ社 (1995)

[5] D. G. Manolakis, V. K. Ingle and S. M. Kogon: Statistical and Adaptive Signal Processing, Artech House (2005)

[6] 和田, 江口, 松本: バイアス補償最小 2 乗法による AR 過程のパラメータ推定について; システム制御情報学会 論文誌, Vol. 2, No. 6, pp. 175-182 (1989)

[7] 和田: 固有べクトル法によるシステム同定について; シ ステム／制御／情報, Vol. 51, No. 1, pp. 34-36 (2007)

[8] T. Söderström: Errors-in-varialbes models in system identification; Automatica, Vol. 43, pp. 939-958 (2007)

[9] S. Beghelli, R. P. Guidorzi and U. Soverini: The Frisch scheme in dynamic system identification; $A u$ - 
tomatica, Vol. 26, pp. 171-176 (1990)

[10] R. Diversi, R. Guidorzi and U. Soverini: A new criterion in EIV identification and filtering applications; 13th IFAC Symposium on System Identification (2003)

\section{著者略歴}

示敖信霍 (正会員)

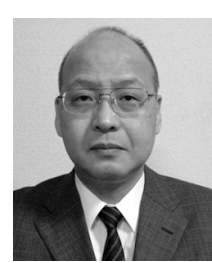

1958 年 8 月 30 日生. 1983 年 3 月大阪 府立大学大学院工学研究科修士課程修了 (航空工学)。同年 4 月日立機電工業（株） (現：(株）日立プラントテクノロジー）入 社，1987 年 9 月日本スピードショア (株) 入社, 1988 年 11 月大阪府立大学工学部航 空宇宙工学科助手, 1996 年 4 月静岡理工科大学理工学部機械 工学科講師, 1999 年 4 月同助教授, 2002 年 4 月大阪工業大学 情報科学部情報科学科助教授（2007 年より准教授）となり現 在に至る. 移動体の位置計測, 誘導に関する研究に従事. 博 士 (工学). 計測自動制御学会, 日本ロボット学会, 日本航 空宇宙学会, 日本機械学会などの会員. 